\title{
LUXACIÓN RECURRENTE DE PATELA
}

\author{
Danny Onzaga MD*, Carlos Eduardo Pardo MD**
}

\section{Resumen}

La luxación de la rótula en su primer episodio es una patología de etiología multifactorial. En la actualidad se encuentran bien identificadas las múltiples causas que tienen relación con este evento. Dentro de ellas no se ha logrado identificar la de mayor importancia en la recidiva de la luxación. El objetivo de este trabajo es caracterizar la importancia de cada uno de estos factores y se inicia con la revisión de la literatura y la descripción e identificación clara de los hallazgos clínicos y paraclínicos. Como es una patología de baja incidencia en la población, buscamos el apoyo interinstitucional para la realización de este trabajo que puede tener un impacto significativo en la decisión terapéutica ante el primer episodio de trauma.

Palabras clave: luxación, patela, rótula, trauma.

\section{RECURRENT PATELLAR DISLOCATION}

\section{Abstract}

The first episode of patellar dislocation has a multi-factorial etiology. Presently the various causes related to this event are well identified. However, the main cause of recurrent dislocation has not been determined. The objective of this work is to categorize the importance of each of these factors and was conducted reviewing relevant literature and describing and clearly identifying clinical and diagnostic findings. As incidence is low for this condition, we sought inter-institutional collaboration for this work which may significantly impact therapeutic alternatives to address the first trauma episode.

Key words: dislocation, patella, trauma.

\section{Introducción}

Se entiende por luxación aguda al primer episodio (por lo regular de origen traumático) en el que se presenta una pérdida de las relaciones articulares donde la patela sale del surco intercondíleo. ${ }^{1,2,3,4}$ La forma recurrente se define como uno o más episodios de luxación de patela en una rodilla que ya había presentado luxación y que no había sido llevada a tratamientos quirúrgicos previos. En términos generales, la reluxación se da por un trauma de alta intensidad sobre las estructuras de la rodilla, ${ }^{5}$ en las que su proceso de reparación fue deficiente impidiendo que cumpla la función de soporte que antes tenía; la mayoría de rodillas con reluxación presentan alteraciones previas (morfológicas, angulares, rotacionales, de potencia muscular o de elasticidad) que predisponen para que un trauma de intensidad menor haga que las estructuras que lograban mantener unas relaciones articulares

Fecha recibido: junio I de 2010 - Fecha aceptado: agosto 10 de 2010

* Residente IV de Ortopedia y Traumatología, Fundación Universitaria de Ciencias de la Salud, Bogotá DC. Colombia.

** Jefe del Servicio de Ortopedia, Hospital de San José. Instructor Asociado, Fundación Universitaria de Ciencias de la Salud, Bogotá DC. Colombia. 
apropiadas, se pierdan y lleven a inestabilidad. La literatura muestra que puede presentarse entre quince ${ }^{6}$ y $44 \%$ de los pacientes ${ }^{7}$ con antecedente de luxación aguda. $\mathrm{Su}$ presentación es más frecuente en mujeres. ${ }^{8}$

Hasta el momento no hay evidencia clara de la historia natural de las luxaciones recurrentes de patela. ${ }^{9}$ Cuando se presentan episodios a repetición el paciente presentará con el tiempo dolor y cambios osteoartrósicos ${ }^{10,11}$ que requieren manejo quirúrgico tanto para controlar el dolor como para disminuir los episodios de luxación, que son angustiantes para el paciente ya que se presentan durante actividades de la vida diaria, a diferencia por ejemplo de inestabilidades como la ruptura del ligamento cruzado anterior que se ven más durante el deporte. ${ }^{12} \mathrm{Se}$ han reportado series en las que quedan con limitaciones para las actividades físicas intensas hasta en el $58 \% .^{13}$

\section{Anatomía}

La patela hace parte de los huesos sesamoideos del cuerpo, descansa sobre el surco troclear en la región intercondílea del fémur, ${ }^{14}$ centraliza y potencia la fuerza del cuadriceps que es el principal músculo extensor de la rodilla. Las características anatómicas de la articulación patelofemoral, así como las fuerzas que en ella actúan, son las que determinan muchos de los diferentes factores que afectan la estabilidad y la función de esta articulación. ${ }^{15}$ Uno de los principales componentes anatómicos está constituido por la forma de la tróclea femoral que es un surco cóncavo formado por un contorno de hueso y cartílago en el cual descansa la patela, la cual tiene una cara articular convexa para relacionarse en contacto con la tróclea femoral convirtiéndose en un restrictor óseo. ${ }^{16}$ Otro componente es la alineación de la extremidad en el que la patela se relaciona con ésta, siendo afectada por la posición en varo-valgo por la alineación tibiofemoral (plano coronal) y el componente rotacional dado por la versión femoral y tibial. El tercer componente corresponde a los tejidos blandos (restrictores pasivos) entre los que se encuentran los ligamentos patelofemoral, patelotibial y patelomeniscal, los cuales tienen relevancia, en especial el patelofemoral medial, ${ }^{1,17}$ al funcionar como bandas de tensión que tratan de mantener la patela en el surco intercondíleo. Un último elemento relevante es la función dinámica del cuadriceps sobre la permanente ubicación dinámica de la patela con respecto al fémur. ${ }^{1,18}$

\section{Factores de riesgo}

Las luxaciones de patela están influenciadas por varios factores que afectan la estabilidad de la articulación de una u otra forma. Entre éstos se encuentran:

1) Edad: la literatura muestra una frecuencia mayor en jóvenes, ${ }^{19-21}$ pero algunos autores tienen opinión contraria. ${ }^{22,23}$ Se ha reportado también la posibilidad de presentar reluxación en quienes presentan el primer episodio en la adolescencia más que siendo adultos jóvenes. ${ }^{24}$

2) Sexo: tiene diferentes aproximaciones, varios trabajos hablan de una mayor incidencia en mujeres entre 58 y $86 \%{ }^{8,25-27}$ Puede deberse al mayor genu valgo o por su hiperlaxitud ligamentaria.

3) Ángulo de congruencia: es el formado por la bisectriz del surco intercondíleo, en donde se considera que la patela es congruente (no lateralizada) cuando la proyección de una línea entre el vértice del ángulo intercondíleo y el punto más posterior de la región articular de la patela se proyecta hacia la bisectriz o hacia adentro de ésta. Cuando su proyección es hacia afuera se considera que se está lateralizando la patela. Esta medida logra mostrar subluxaciones mínimas que ya se presentan en los que sufren de inestabilidad patelofemora ${ }^{28}$ (Figura 1).

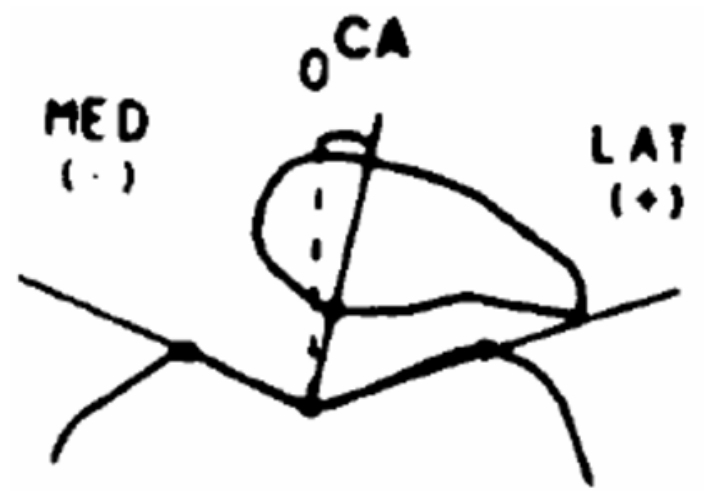

Figura I. Ángulo de congruencia. Tomado de Kujala U, Osterman K, Kormano. Patellofemoral relationships in recurrent patelar dislocation. J Bone Joint Surg (Br) 1989; 7 IB: 788-92. 
4) Mal alineamiento femoropatelar: se puede valorar con el ángulo Q que está en una línea imaginaria entre la espina iliaca anterosuperior, el punto central de la patela y el centro de la tuberosidad anterior de la tibia. Cuando la rodilla se encuentra a $30^{\circ}$ de flexión, este ángulo debe encontrarse en un rango entre $8^{\circ}$ y $10^{\circ}$ para hombre y $15^{\circ} \pm 5^{\circ}$ en mujeres. Esta medida muestra cómo está la ubicación de la patela respecto a la alineación en el plano coronal que se encuentra en la extremidad inferior. Debe tenerse en mente que este ángulo $Q$ es la expresión de la alineación de la extremidad, pero puede estar aumentado por múltiples condiciones como anteversión del cuello femoral, genu valgo, torsión tibial externa o un retináculo lateral tirante..$^{20,29}$

5) Genu varo-valgo: los pacientes con genu valgo tienen más posibilidad de presentar luxaciones laterales de patela. Se define como el ángulo que forma el eje del fémur respecto al de la tibia, el cual debe ser $6^{\circ}+/-1^{\circ}$. Ángulos mayores de $7^{\circ}$ se consideran genu valgo y menores de $5^{\circ}$ son varos.

6) Ángulo del surco intercondileo: se entiende como el existente entre la punta anterior de cada cóndilo y el punto más profundo del surco intercondíleo. Este ángulo se ha visto aumentado cuando se presenta displasia del mecanismo extensor, ${ }^{30}$ lo que quiere decir que el surco intercondíleo femoral está más aplanado y así la patela pierda el soporte óseo que debe brindar a cada uno de sus lados. También es importante la ubicación del surco intercondíleo respecto a la perpendicular al plano coronal, teniendo mayor estabilidad y menor propensión a la luxación de los surcos que se encuentran más laterales ${ }^{31}$ (Figura 2).

7) Tipos de contorno patelar: la estabilidad ósea de la articulación patelofemoral depende en parte de la relación contenido-continente en el cual la arista formada por la patela case en forma congruente con el surco intercondíleo. Se da inestabilidad cuando la patela no tiene la forma correspondiente al surco. Estas diferencias fueron descritas por Wiberg. Tener una patela alejada de la forma usual en las cua- les sus caras articulares no son cóncavas e idénticas, aumenta la inestabilidad ósea entre el fémur y la patela $^{32,33}$ (Figura 3).

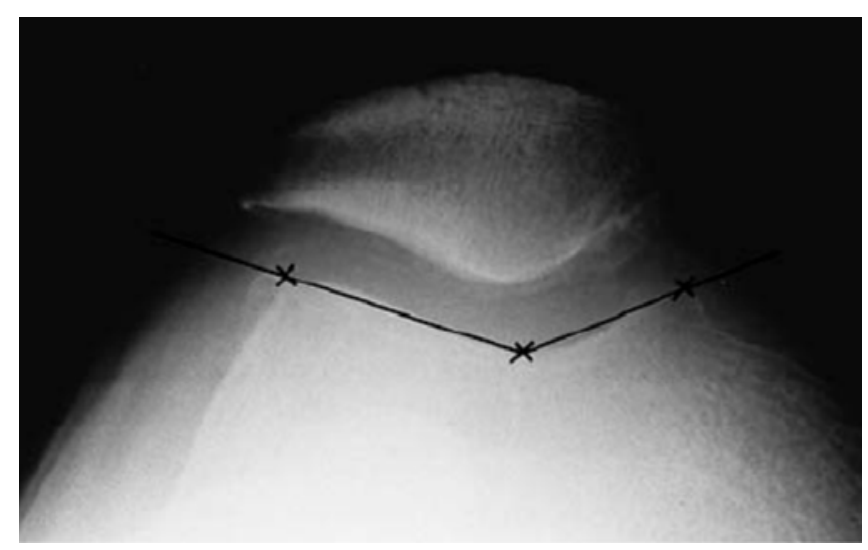

Figura 2. Ángulo del surco intercondíleo. Tomado de P. Davies ML, Costa ST, Donnell MM, Glasgow and L. Shepstone. The sulcus angle and malalignment of the extensor mechanism of the knee. J Bone Joint Surg Br, Nov 2000; 82-B: I I62-I I66.

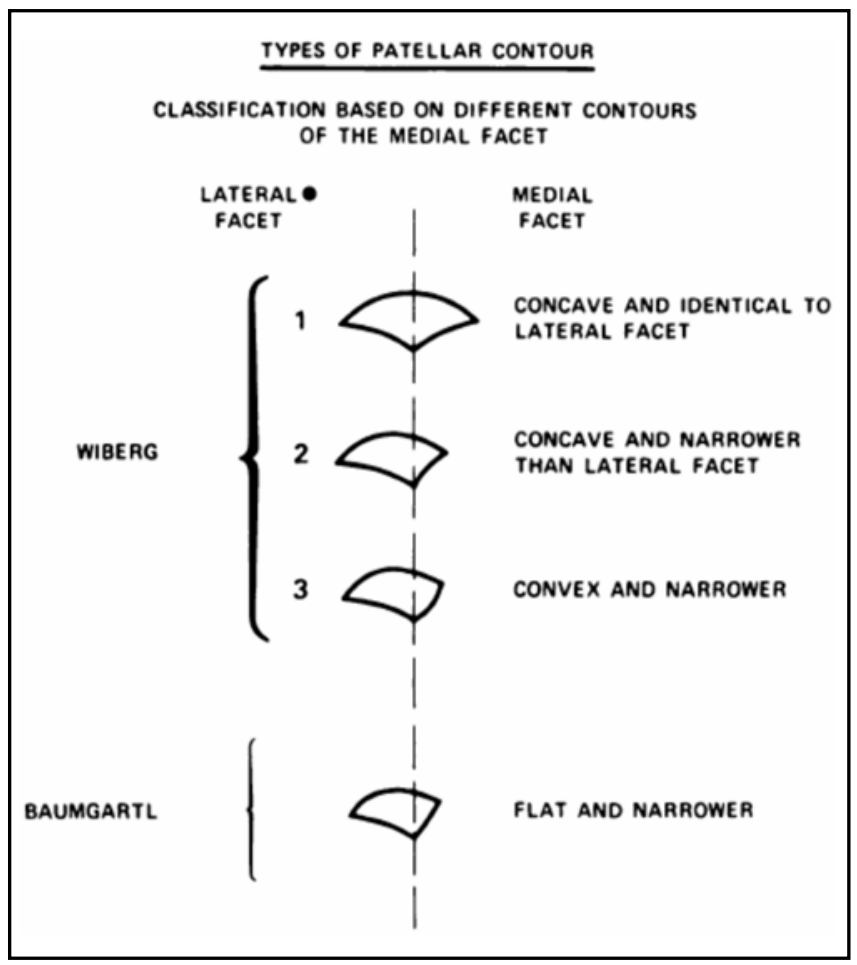

Figura 3. Tipos de contorno patelar. Tomado de Laurin CA, Lévesque HP, Dussault R, Labelle H, Peides JP. The abnormal lateral patellofemoral angle: a diagnostic roentgenographic sign of recurrent patellar subluxation. J Bone Joint Surg (Am) 1978; 60 (I): 55-60. 
8) Anteversión femoral: se refiere al ángulo formado por la inclinación anterior que tiene el cuello femoral respecto a una línea intercondílea (Figura 4). Una forma de medirlo en la clínica diaria es teniendo al paciente en decúbito prono, se realiza una rotación interna de la extremidad en estudio mientras está con flexión de $90^{\circ}$ de la rodilla, y con una mano se palpa simultáneamente la región trocantérica hasta encontrar el punto en el cual la prominencia trocantérica está más externa. Se considera la perpendicular al piso y el eje de la tibia para así formar el ángulo de versión del cuello femoral. ${ }^{33}$ Cuando la anteversión femoral está aumentada hace que el fémur adopte una posición en rotación interna ocasionando que el tendón patelar fijo a la tuberosidad traccione envía lateral la patela, produciendo una fuerza mayor a la que puede contrarrestar el músculo oblicuo medio y favorece por lo tanto la luxación de la patela (Figura 5). ${ }^{33}$

9) Patela alta: es la relación de la ubicación de la patela entre la tróclea femoral en sentido cefálico y la interlínea articular. Se puede volver objetiva con mediciones como el índice de Insall, que relaciona la lon-

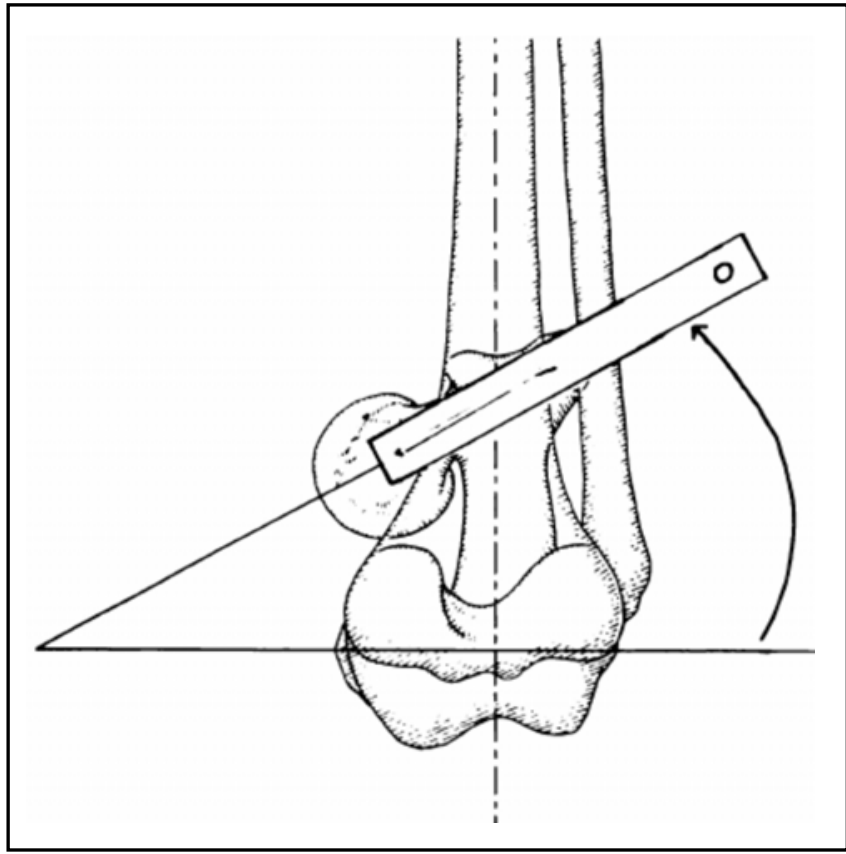

Figura 4. Ángulo de anteversión femoral. Tomado de Ruwe PA, Gage JR, Ozonoff MB, deLuca PA. Clinical determination of femoral anteversion. J Bone Joint Surg (Am) 1992; 74 (6): 820-30.

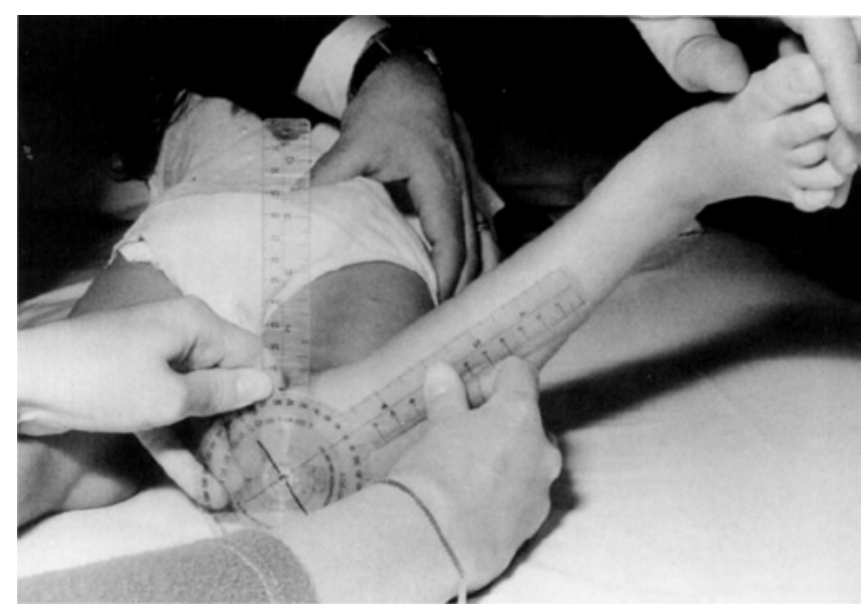

Figura 5. Ángulo de anteversión femoral. Tomado de Ruwe PA, Gage JR, Ozonoff MB, deLuca PA. Clinical determination of femoral anteversion. J Bone Joint Surg (Am) 1992; 74 (6): 820-30.

gitud entre el borde superior de la punta superior y la punta inferior de la patela sobre la longitud entre el polo inferior de la patela y la parte más superior de la tuberosidad anterior de la tibia. Se considera una patela alta cuando este índice es igual o mayor a 1.3. Al estar la patela ascendida respecto a las relaciones que debería tener con el surco intercondíleo, la patela pierda la estabilidad ósea ofrecida por el fémur, facilitando su luxación (Figura 6).

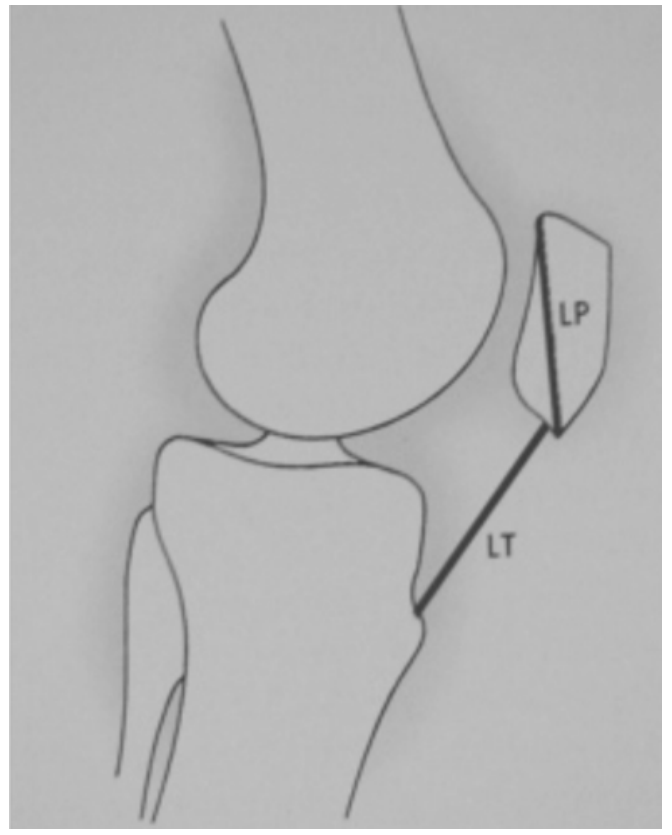

Figura 6. Índice de Insall. Tomado de Canale S, Beaty J. Campbell's Operative Orthopaedics. Mosby; (IV): 2658. 
10) Ángulo patelofemoral lateral: la mayoría de rodillas consideradas normales tienen el ángulo abierto hacia la región lateral formado por los puntos más anteriores del surco intercondíleo y los de la carilla articular lateral (Figura 7). ${ }^{32}$ Se considera que la inclinación del ángulo patelofemoral lateral no debe ser paralelo o de ángulo abierto interno. Este hallazgo habla de la displasia del cuadriceps, ${ }^{30}$ lo que hace que la patela se "traccione" hacia la zona lateral cuando hay displasia o disminución de la potencia del vasto interno, lo que lleva a las estructuras laterales de la rodilla (vasto externo, retináculo) a dominar este balance de fuerzas, lo que predispone a que la patela se lateralice y subluxe.

11) Hiperlaxitud ligamentaria: cuando los ligamentos son muy laxos pierden un porcentaje de su capacidad como restrictores permitiendo rangos de movilidad excesivos de la patela respecto a la extremidad. Este estado de hiperlaxitud se puede medir en la práctica médica ${ }^{34}$ por medio de signos clínicos que se encuentran al examen físico como hiperdorsiflexión

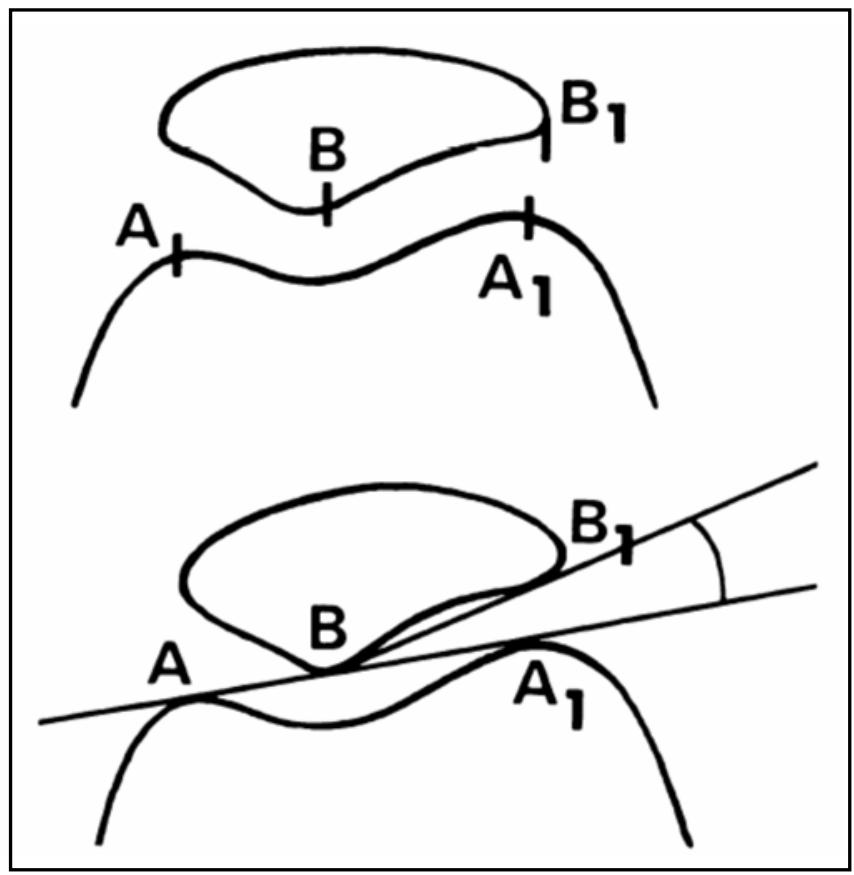

Figura 7. Ángulo patelofemoral lateral. Tomado de Laurin CA, Lévesque HP, Dussault R, Labelle H, Peides JP. The abnormal lateral patellofemoral angle: a diagnostic roentgenographic sign of recurrent patellar subluxation. J Bone Joint Surg (Am) 1978; 60 (I): 55-60. del pie o hiperextensión de las articulaciones metacarpofalángicas.

\section{Tratamiento}

El manejo de la luxación recurrente de patela, sigue siendo un punto de discusión. ${ }^{11,35}$ Prueba de ello está en las múltiples técnicas quirúrgicas para su corrección que se encuentran en la literatura médica, lo que puede indicar que muchas veces los resultados no son los mejores, logrando disminuir el riesgo de reluxación pero no los síntomas secundarios, ${ }^{12,24,36,37} \mathrm{e}$ incluso con resultados objetivos mejores en el manejo ortopédico que en el quirúrgico. ${ }^{20}$

Cuando se analiza el tratamiento ortopédico se aprecia un consenso en la recomendación de manejar el primer episodio en forma médica (terapia física,${ }^{38}$ inmovilización y AINEs), ${ }^{17,28,39-41}$ pero al momento de presentar la reluxación parece ser insuficiente. ${ }^{24,40}$ Dentro del enfoque para realizar tratamiento quirúrgico en luxación aguda de patela se recomienda que todos aquellos los que presentan hemartrosis deben llevarse a rayos $\mathrm{X}$ y resonancia magnética en busca de fracturas osteocondrales susceptibles de fijación en el período agudo, o bien cuerpos libres que puedan causar síntomas. ${ }^{26,39}$ Pero en la luxación recurrente no hay un esquema claro de las opciones terapéuticas. Los esfuerzos quirúrgicos están encaminados a modificar aquellos factores que puedan ser los causantes de la posición anómala de la patela. ${ }^{12,40}$ Por lo general se clasifican como procedimientos proximales (reconstrucción de ligamento patelofemoral medial, liberación del retináculo lateral ${ }^{42,43}$ y trocleoplastias) y distales (osteotomía de Fulkerson, Emslie-Trillat, Roux Goldthwaite) que en términos generales modifican la inserción del tendón patelar). ${ }^{17,40,44,45}$ Se recomiendan los procedimientos distales cuando se encuentra un verdadero mal recorrido de la patela por el surco intercondíleo, ${ }^{17}$ que se diagnostica mediante TAC de patela a diferentes grados de flexión. Los procedimientos proximales como la reconstrucción del ligamento patelofemoral medial se reservan para aquellos casos que por la clínica o RM revelan insuficiencia de este ligamento. Una consideración interesante sobre la indicación o no de repararlo se puede inferir a partir del 
estudio realizado por Fithian, ${ }^{8}$ quien encuentra que en pacientes con luxación aguda de patela con ruptura del ligamento patelofemoral medial tienen menor incidencia de reluxación; esto podría explicarse si se tiene en cuenta que las luxaciones de patela sin lesión del ligamento patelofemoral medial, pueden indicar que coexiste una displasia patelofemoral. Schöttle ${ }^{46}$ muestra los resultados de una pequeña serie observando buenos resultados en los parámetros radiológicos y en las escalas subjetivas mediante la corrección de este ligamento, que también Nomura refiere en su serie de casos. ${ }^{47}$ En una revisión sistemática Smith y col. ${ }^{48}$ valoraron la información anterior encontrando vacíos metodológicos que obligan a mirar con discreción los resultados publicados. El estudio de Sillanpää ${ }^{49}$ compara los resultados a largo plazo entre intervención proximal (reconstrucción del ligamento patelofemoral medial) e intervención distal (RouxGoldthwait), encontrando similares resultados en la incidencia de reluxación en los dos grupos pero con mayor incidencia de osteoartrosis en el de corrección distal. Respecto a la trocleoplastia, se recomienda en aquellos pacientes con el surco intercondíleo muy aplanado o prominente, ${ }^{36,44}$ pero los resultados de este procedimiento muestran una mejoría apenas aceptable.

Al momento de escoger cuál será el tratamiento quirúrgico se debe individualizar cada caso, observando cuáles de los factores antes descritos son los que predominan, lo que obliga a tener una serie de ayudas paraclínicas como radiografías, TAC, resonancia magnética, para lograr tener un panorama completo y objetivo. ${ }^{50}$ Andrich ${ }^{12}$ recomienda realizar los procedimientos quirúrgicos (en la medida de lo posible) bajo anestesia local o con regional periférica, con el fin de permitir la extensión activa de la rodilla, sobre todo para los que presentan luxación al extender la rodilla o que tienen signos de la J prominentes.

\section{Conclusión}

La luxación recurrente de patela sigue siendo un tema que requiere amplia investigación, donde hay múltiples variables anatómicas y demográficas que se interrelacionan, obligando al cirujano a identificarlas, valorarlas y a llegar a un planteamiento médico o quirúrgi- co, que muchas veces necesita de varias etapas y de la utilización de diferentes técnicas operatorias, que deben enfocarse a la corrección anatómica, en forma diferente por cada paciente, convirtiendo en un reto el control de los síntomas y la prevención de las secuelas.

\section{Referencias}

1. Arendt EA, Fithian DC, Cohen E. Current concepts of lateral patella dislocation. Clin Sports Med. 2002;21(3):499-519

2. Kapur S. Wissman RD, Robertson M, Verma S, Kreeger MC, Oostveen RJ Acute knee dislocation: review of an elusive entity. Curr Probl Diagn Radiol 2009;38(6): 237-50

3. Nikku R. Nietosvaara Y, Aalto K, Kallio PE. The mechanism of primary patellar dislocation: trauma history of 126 patients. Acta Orthop. 2009;80(4): $432-4$

4. Nietosvaara Y, Paukku R, Palmu S, Donell ST. Acute patellar dislocation in children and adolescents. Surgical technique. J Bone Joint Surg Am. 2009;91 Suppl 2 (Pt 1):139-45

5. Phillips BB. Patellar dislocation. En: Daugherty K, Jones L, editors. Campbell's Operative Orthopaedics. 11 ed. Philadelphia: Mosby; 2008. p 2655-72.

6. Macnab I. Recurrent dislocation of the patella. J Bone Joint Surg Am. 1952 Oct;34 A(4): 957-67.

7. Stefancin JJ, Parker RD. First-time traumatic patellar dislocation: a systematic review. Clin Orthop Relat Res. 2007;455:93-101

8. Fithian DC, Paxton EW, Stone ML, Silva P, Davis DK; Elias DA, et al. Epidemiology and natural history of acute patellar dislocation. Am J Sports Med. 2004;32(5): 1114-21

9. White BJ, Sherman OH. Patellofemoral instability. Bull NYU Hosp Jt Dis 2009;67(1): 22-9

10. Maenpaa H, Lehto MU. Patellofemoral osteoarthritis after patellar dislocation Clin Orthop Relat Res 1997;339:156-62

11. Arnbjornsson A, Egund N, Rydling O, Stockerup R, Ryd L. The natural history of recurrent dislocation of the patella. Long-term results of conservative and operative tratment. J Bone Joint Surg Br. 1992;74(1):140-2

12. Andrish J. The management of recurrent patellar disolocation. Orthop Clin North Am. 2008:39(3):313-27

13. Atkin DM, Fithian DC, Marangi KS ML, Dobson BE, Mendelsohn C. Characteristics of patients with primary acute lateral patella dislocation and their recovery within the first 6 months of injury. Am J Sports Med. 2000; 28(4):472-9.

14. Hinton RY, Sharma KM. Acute and recurrent patellar instability in the young athlete. Orthop Clin North Am. 2003;34(3):385-9.

15. Fithian DC, Paxton EW, Cohen AB. Indications in the treatment of patellar instability. J Knee Surg. 2004;17(1):47-56

16. Arendt E. Anatomy and malalignment of the patellofemoral joint: its relation to patellofemoral arthrosis. Clin Orthop Relat Res. 2005;(436):71-5. 
17. Lim AK, Chang HC, Hui JH. Recurrent patellar dislocation: reappraising our approach to surgery. Ann Acad Med Singapore. 2008;37(4):320-3.

18. Floyd A, Phillips P, Khan MR, Webb JN, McInnes A, Hughes SP. Recurrent dislocation of the patella. Histochemical and electromyographic evidence of primary muscle pathology. J Bone Joint Surg Br. 1987;69(5):790-3.

19. Cash JD, Hughston JC. Treatment of acute patellar dislocation. Am J Sports Med. 1988;16(3):244-9.

20. Nikku R, Nietosvaara Y, Aalto K, Kallio PE. Operative treatment of primary patellar dislocation does not improve medium-term outcome: A 7-year followup report and risk analysis of 127 randomized patients. Acta Orthop. 2005; 76(5):699-704

21. Runow A. The dislocating patella. Etiology and prognosis in relation to generalized joint laxity and anatomy of the patellar articulation. Acta Orthop Scand Suppl. 1983;201:1-53

22. Cofield RH, Bryan RS. Acute dislocation of the patella: results of conservative treatment. J Trauma. 1977; 17(7):526-31

23. Weber-Spickschen TS, Spang J, Kohn L. Imhoff AB, Schottle PB. The relationship between trochlear dysplasia and medial patellofemoral ligament rupture location after patellar dislocation: An MRI evaluation. Knee. 2010 Publicación electrónica 2010 May 20

24. Larsen E, Lauridsen F. Conservative treatment of patellar dislocations. Influence of evident factors on the tendency to redislocation and the therapeutic result Clin Orthop Relat Res. 1982;171:131-6.

25. Maenpaa H, Lehto MU. Surgery in acute patellar dislocation - evaluation of the effect of injury mechanism and family occurrence on the outcome of treatment. Br J Sports Med. 1995;29(4):239-41

26. Kettelkamp DB. Management of patellar malalignment. J Bone Joint Surg Am. 1981;63(8):1344-8

27. Hughston JC. Subluxation of the patella. J Bone Joint Surg Am 1968;50(5):1003-26

28. Kujala UM, Osterman K, Kormano M, Nelimarkka O, Hurme M, Taimela S Patellofemoral relationships in recurrent patellar dislocation. J Bone Joint Surg Br. 1989;71(5):788-92.

29. Sarkar A. Razdan S, Yadav J, Bansal N, Kuhar S, Pahuja P. Indian J Physiol Pharmacol. 2009;53(3):275-8.

30. Davies AP, Costa ML, Shepstone L, Glasgow MM, Donell S. The sulcus angle and malalignment of the extensor mechanism of the knee. J Bone Joint Surg Br. 2000;82(8):1162-6

31. Hing CB, Shepstone L, Marshall T, Donell ST. A laterally positioned concave trochlear groove prevents patellar dislocation. Clin Orthop Relat Res. 2006;447:187-94

32. Laurin CA, Levesque HP, Dussault R, Labelle H, Peides JP. The abnormal lateral patellofemoral angle: a diagnostic roentgenographic sign of recurrent patellar subluxation. J Bone Joint Surg Am. 1978;60(1):55-60.

33. Ruwe PA, Gage JR, Ozonoff MB, DeLuca PA. Clinical determination of femoral anteversion. A comparison with established techniques. J Bone Joint Surg Am. 1992;74(6):820-30.
34. Carter C, Wilkinson J. Persistent joint laxity and congenital dislocation of the hip. J Bone Joint Surg Br. 1964;46:40-5

35. Camanho GL, Viegas Ade C, Bitar AC, Demange MK, Hernandez AJ. Arthroscopy. 2009;25(6):620-5.

36. Verdonk R, Jansegers E, Stuyts B. Trochleoplasty in dysplastic knee trochlea. Knee Surg Sports Traumatol Arthrosc. 2005;13(7):529-33.

37. Aglietti P, Buzzi R, De BP, Giron F. Surgical treatment of recurrent dislocation of the patella. Clin Orthop Relat Res. 1994;308:8-17.

38. Bhave A, Baker E. Prescribing quality patellofemoral rehabilitation before advocating operative care. Orthop Clin North Am. 2008;39(3):275-85

39. Mehta VM, Inoue M, Nomura E, Fithian DC. An algorithm guiding the evaluation and treatment of acute primary patellar dislocations. Sports Med Arthrosc. 2007;15(2):78-81

40. Mulford JS, Wakeley CJ, Eldridge JD. Assessment and management of chronic patellofemoral instability. J Bone Joint Surg Br. 2007;89(6):709-16.

41. Von Engelhardt LV. Raddatz M. Bouilon B, Spahn G, Dávid A, Haage P, Lichtinger TK. How reliable is MRI in diagnosing cartilaginous lesions in patients with first and recurrent lateral patellar dislocations?. BMC Musculoskelet Disord. 2010;11:149

42. Dandy DJ, Griffiths D. Lateral release for recurrent dislocation of the patella. J Bone Joint Surg Br. 1989;71(1):121-5.

43. Akgün U, Nuran R, Karahan M. Modified Fulkerson osteotomy in recurrent patellofemoral dislocations. Acta Orthop Traumatol Turc. 2010;44(1):27-35

44. Donell ST, Joseph G, Hing CB, Marshall TJ. Modified Dejour trochleoplasty for severe dysplasia: operative technique and early clinical results. Knee. 2006;13(4):266-73.

45. Dannawi Z, Khanduja V, Palmer CR, El-Zebdeh M. Evaluation of the modified Elmslie-Trillat procedure for patellofemoral dysfunction. Orthopedics. 2010;33(1):13.

46. Schottle PB, Fucentese SF, Romero J. Clinical and radiological outcome of medial patellofemoral ligament reconstruction with a semitendinosus autograft for patella instability. Knee Surg Sports Traumatol Arthrosc. 2005;13(7):516-21

47. Nomura E, Inoue M, Kobayashi S. Long-term follow-up and knee osteoarthritis change after medial patellofemoral ligament reconstruction for recurrent patellar dislocation. Am J Sports Med. 2007;35(11):1851-8.

48. Smith TO, Walker J, Russell N. Outcomes of medial patellofemoral ligament reconstruction for patellar instability: a systematic review. Knee Surg Sports Traumatol Arthrosc. 2007;15(11):1301-14

49. Sillanpaa P. Mattila VM, Visuri T, Maenpaa H, Pihlajamaki H. Ligament reconstrucction versus distal realifnment for patellar dislocation. Clin Orthop Relat Res. 2008;466(6): 1475-84.

50. Han H, Xia Y, Yun X, Wu M. Anatomical transverse patella double tunnel reconstruction of medial patellofemoral ligament with a hamstring tendon autograft for recurrent patellar dislocation. Arch Orthop Trauma Surg. 2010 Publicación electrónica 2010 Aug 17. 